\title{
Monotherapy in serious hospital-acquired infections: a clinical trial of ceftazidime versus imipenem/cilastatin
}

\author{
S. Ragnar Norrby**, Roger G. Finch*, Michel Glauser ${ }^{c}$, and a European Study \\ Group**
}

${ }^{a}$ Department of Infectious Diseases, University of Lund, Sweden; ${ }^{b}$ Department of Microbial Diseases, The City Hospital and University of Nottingham, UK; 'Division of Infectious Diseases, Department of Medicine, CHUV University of Lausanne, Lausanne, Switzerland

\begin{abstract}
The clinical and bacteriological efficacy and safety of the antibiotics ceftazidime or imipenem/cilastatin in seriously ill patients with nosocomial infections were compared in a prospective, open, evaluator-blind, multicentre comparative trial. The study was performed in 26 European centres, the majority being intensive care units. Subjects were randomized to receive either ceftazidime $2 \mathrm{~g}$ bid or imipenem cilastatin $0.5 \mathrm{~g}$ qid given for at least five days after stratification for pneumonia, septicaemia or urinary tract infection (UTI). Three hundred and ninety-three patients with serious nosocomial infections (254 with pneumonia; 91 with septicaemia and 48 UTI were treated between February 1988 and January 1990 and their clinical and bacteriological response to antibiotic treatment assessed. There were no significant differences between ceftazidime and imipenem/cilastatin in clinical efficacy. The failure rates in evaluable patients were 22 and $26 \%$ in pneumonia, 23 and $19 \%$ in septicaemia and 0 and $5 \%$ respectively in those with UTI. Overall there was no significant difference between the two antibiotics for bacteriological response in the three infection strata. However, in patients with pneumonia ceftazidime was significantly more effective than imipenem/cilastatin in clearing patients of
\end{abstract}

\footnotetext{
- Correspondence to: Professor S. R. Norrby, Department of Infectious Diseases, Lund University Hospital, S-22185 Lund, Sweden.

**Members of the study group (no. of patients entered) were W. Graninger (41), Universitätsklinik für Chemotherapie, Vienna, Austria; H. Hüneburg and C. Knorr (36), St. Petrus Krankenhaus, Bonn, Germany; P. Eggiman and A. Cometta (37), CHU Vaudois, Lausanne, Switzerland; D. Adelt (33), Klinikum der RWHT Aachen, Aachen, Germany; X. Garau (27), Mutua de Tarassa Hospital, Barcelona, Spain; A. Bossaert, L. G. Heytens and S. R. Pattyn (24), UZA,. Edegem, Belgium; M. Fischer and H. Nusser (20), Stadt Krankenhaus, Passau, Germany; A. Friesen and A. Schilling (19), Stadt KH Bogenhausen, Munich, Germany; J. D. Edwards, A. Mortimer and B. Oppenheim (17), Withington Hospital, Manchester, UK; B. Hill, J. Norgren and A. Rydvall (15), Sundsvall Hospital, Sundsvall, Sweden; G. B:son Asklö, C. Hansson and T. Nolin (15), Kristianstad Hospital, Kristianstad, Sweden; A. Porto and A. S. P. De Carvahlo (14), Hospitais da Universidade de Coimbra, Portugal; C. C. Smith, T. M. S. Reid and I. M. Gould (14), City Hospital, Aberdeen, UK; D. Rimmer and A. B. Knight-Guilhem (14), Hillingdon Hospital, Uxbridge, UK; K. Alestig, R. Andersson and S. Arvidsson (13), Ostra Hospital, Gothenburg, Sweden; P. O. Eklund, C. Nilsson and R. Neringer (11), Karlskrona Hospital, Karlskrona, Sweden; G. Klein (10), Johann W. Göthe University Frankfurt, Frankfurt am Main, Germany; K. Allen and R. R. Macmillan (9), Whiston Hospital, Merseyside, UK; H. Lessire and N. Puchstein (7), Katholisches Krankenhaus, Herne, Germany; H. Mittermayer and L. Binder (6), A. O. KH der Elisabethineu, Linz, Austria; K. Sammalkorpi (3), Aurora Hospital, Helsinki, Finland; G. Verger (3), Hospital Santa Cruz y San Pau, Barcelona, Spain; J. F. Bion (2), Queen Elizabeth Hospital, Birmingham, UK; C. Brun-Buisson (1), Hospital Henri Monder, Creteil, France; G. Lindsay (1), Southern General Hospital, Glasgow, UK; F. K. Gould (1), Freeman Hospital, Newcastle upon Tyne, UK.
} 
Pseudomonas spp.: $3 / 17$ and $11 / 19$ patients respectively had persistent growth of Pseudomonas spp. post-treatment $(P=0004)$, and in one ceftazidime failure resistance emerged compared to six imipenem/cilastatin failures in which resistance emerged. Few drug-related adverse events were recorded in either treatment group. Monotherapy with either ceftazidime ( $2 \mathrm{~g}$ bid) or imipenem/cilastatin $(0.5 \mathrm{~g}$ qid) is safe and effective and could be considered as an alternative to combination therapy for the treatment of serious hospital-acquired infections.

\section{Introduction}

Hospital-acquired infections are common and may be life-threatening. The most serious are pneumonia and septicaemia, which may originate from infectious foci in the lungs, the urinary tract or other sites. Nosocomial pneumonia presents a particular problem, especially in patients requiring assisted ventilation. In such patients colonization of the oropharynx with multiresistant hospital pathogens can lead, in the absence of mechanical or local defences, to a very high incidence of nosocomial pneumonia, as shown in several recent studies (Stoutenbeek et al., 1987; Ledingham et al., 1988). Aerobic Gram-positive or Gram-negative bacterial pathogens are the most common causative agents. Anaerobes may be important in patients who have developed pneumonia after aspiration of gastric contents and in those with lung abscesses or empyema (Carpenter, 1990).

The treatment of nosocomial infections should cover the most common aerobic bacterial species, including Staphylococcus aureus and Pseudomonas aeruginosa. Previously this could only be achieved with combination antibiotic therapy, usually an aminoglyoside with a broad spectrum $\beta$-lactam. However, a clear drawback to the use of aminoglycosides in patients with pneumonia is the need for high doses in order to achieve an optimal therapeutic outcome (Moore, Smith \& Lietman, 1984). This may be due to the $\mathrm{pH}$ of bronchial secretions, which is considerably below the optimum $\mathrm{pH}$ for aminoglycoside antibacterial activity (Laforce, 1989).

Recently the development of potent parenteral antibiotics with broad antibacterial activity has offered the potential use of monotherapy for the treatment of serious hospital-acquired infection (Gardner, 1983; Laforce, 1989). Such monotherapy would reduce the treatment costs, and possibly also the risk of adverse reactions from combinations of antibiotics.

This study was undertaken to compare the efficacy of two broad-spectrum antibiotics, ceftazidime and imipenem/cilastatin in the empirical treatment of serious infections developing in patients hospitalized for at least $48 \mathrm{~h}$. Ceftazidime and imipenem/cilastatin both cover a wide range of nosocomial pathogens, and have been extensively used as monotherapy in serious infections, including the treatment of sepsis in immunodeficient patients (Clissold, Todd \& Campoli-Richards, 1987; Sanders, Powe $\&$ Moore, 1991). The notable feature of ceftazidime is its activity against $P$. aeruginosa, while imipenem/cilastatin has greater activity than third generation cephalosporins against staphylococci and anaerobes.

\section{Methods}

The study was undertaken in 26 European centres between February 1988 and January 1990. The protocol was approved by the Ethics Committee in each participating centre, and informed consent, either verbal or written, was requested from the patient or a relative before entering the trial. 


\section{Trial design}

This was a prospective, open, evaluator-blind, multicentre comparative trial. Patients were randomized by the use of sealed, numbered envelopes. Allocation to treatment was in blocks of six, and within each bloc, three patients received each treatment. Stratification was made for pneumonia, septicaemia, or UTI.

\section{Sample size}

Sample size was determined based on the assumption that the expected clinical response rate (cure and improvement) for ceftazidime and imipenem/cilastatin was approximately $85 \%$ at the post-treatment visit. Thus approximately 160 clinically evaluable patients were required in each treatment group to detect a difference in response rate of $10 \%$ with $80 \%$ power using a $5 \%$ level of significance.

\section{Patients}

Hospitalized adult patients ( $\geqslant 16$ years) who developed clinical signs of pneumonia, septicaemia or severe UTI 48 h or more after hospital admission were eligible. Patients who had received previous antibiotic treatment were included only if they had failed to improve or the relevant pathogen had not been eliminated. As a measure of severity of illness, APACHE II scores were calculated in most patients at entry (Knaus et al., 1985). Clinical pneumonia was defined as a new or progressive pulmonary infiltrate on chest X-ray without a known non-infectious cause but with negative or unobtainable bacteriological investigation. In patients with bacteriologically verified pneumonia, organisms considered by the monitoring committee to be clinically relevant must have been isolated from purulent sputum, bronchial secretion, bronchial washing, lung tissue or blood culture. Clinical septicaemia was defined as clinical deterioration and rigors in association with unstable haemodynamic parameters and/or coagulopathy consistent with sepsis in the absence of positive blood cultures. Patients with a bacteriologically documented septicaemia had one or more positive blood cultures, although blood cultures which grew coagulase-negative staphylococci or diphtheroids in only one bottle were considered to be contaminated. Severe UTI included acute pyelonephritis (fever associated with loin pain or tenderness and associated with significant bacteriuria, namely, $\geqslant 10^{7} \mathrm{cfu} / \mathrm{L}$ urine) or complicated UTI (significant bacteriuria in a systemically unwell patient with a temperature $>38.5^{\circ} \mathrm{C}$ and structural or functional abnormalities of the urinary tract, known to predispose to, or permit persistance of, infection). Exclusion criteria for study entry were hypersensitivity to cephalosporins, carbapenems or penicillins, neutropenia $\left(<1.0 \times 10^{9} \mathrm{~L}\right.$ ), likelihood of death within $24 \mathrm{~h}$, isolation before therapy of pathogens known or suspected to be resistant to either of the study drugs, therapy with ceftazidime or imipenem/cilastatin within the previous four weeks, and previous entry into the study.

\section{Antibiotic treatment}

Ceftazidime (Glaxo) was administered in $2 \mathrm{~g}$ doses every $12 \mathrm{~h}$ as bolus intravenous injections over 5-10 min or as intermittent infusions over 20-30 min. Imipenem/cilastatin (Merck Sharp \& Dohme) was given in doses of $500 \mathrm{mg}$ every $6 \mathrm{~h}$ as intermittent infusions over $30 \mathrm{~min}$. With both drugs, doses were reduced in patients with compromised renal function in accordance with the recommendations of the manufacturers. 


\section{Assessment of response}

Clinical response was assessed as cure (clinical symptoms and signs subsided with complete resolution of active infection during the treatment period) or failure (change of antibiotic therapy because of initial isolation of a resistant organism, addition of a new antibacterial agents at any time during treatment, because of clinical deterioration or superinfection at the primary site of infection, and death due to infection). In addition, for pneumonia and severe UTI a category of improvement (clinical signs and symptoms subsided but with incomplete resolution of active infection during treatment) was allowed. Clinical relapse was defined as recurrence of clinical signs and symptoms of the initial infection during the follow-up period, after initial cure or improvement.

Bacteriological response was assessed $24-48 \mathrm{~h}$ after the end of treatment in patients with pneumonia or septicaemia and five to nine days after treatement in those with UTI. It was classified as clearance (absence of the original pathogen(s) in a posttreatment sample or unavailability of such sample in a clinically cured patient), partial clearance (at least one of multiple pathogens eliminated in the post-treatment sample), or failure (continued isolation of the original pathogen(s) at the end of treatment). In patients with UTI repeat urine samples were obtained four to six weeks post-treatment. Isolation of a new organism at the initial site of infection during treatment, or within two days after treatment, was classified as colonization if no further treatment was required and superinfection if treatment was started.

\section{Susceptibility testing}

All relevant organisms isolated at each of the participating centres were tested for susceptibility to ceftazidime and imipenem/cilastatin by a disc diffusion method (either Kirby-Bauer $(70 \%$ of centres) or Stokes (30\% of centres)). Susceptibility testing was carried out using imipenem/cilastatin $(10 \mu \mathrm{g})$ and ceftazidime $(30 \mu \mathrm{g})$ discs on DST, Iso-Sensitest or Mueller-Hinton agars (Oxoid, BBL). Isolates were categorized as susceptible, intermediate or resistant to ceftazidime and imipenem/cilastatin according to the criteria laid down by the National Committee for Clinical Laboratory Standards (NCCLS) in 1987. In addition, the isolates were also sent to a central laboratory for confirmation of identity and minimum inhibitory concentration (MIC) determination. MICs were determined on solid medium (Iso-Sensitest, Oxoid), and interpreted according to the NCCLS (1987) guidelines: ceftazidime-susceptible ( $\leqslant 8 \mathrm{mg} / \mathrm{L}$ ) ceftazidime-intermediate $(16 \mathrm{mg} / \mathrm{L})$, ceftazidime-resistant $(\geqslant 32 \mathrm{mg} / \mathrm{L})$, imipenem/cilastatinsusceptible $(\leqslant 4 \mathrm{mg} / \mathrm{L})$, imipenem/cilastatin-intermediate $(8 \mathrm{mg} / \mathrm{L})$, imipenem/cilastatin-resistant ( $\geqslant 16 \mathrm{mg} / \mathrm{L})$.

\section{Adverse event monitoring}

The investigators were asked to register all unexpected clinical events during or within 14 days after treatment.

\section{Patient evaluation}

Case record forms were filled out by the investigators, screened for accuracy by a monitor and evaluated by one of the members of the monitoring committee (S.R.N.), who was blinded to treatment allocation. The blinded evaluator assessed the clinical and bacteriological responses, and reviewed adverse events. Cases in which the assess- 
ments of the investigators were changed were discussed by all members of the monitoring committee (S.R.N., R.G.F. and M.G.).

\section{Statistical analysis}

The treatment groups were compared within each stratum, with respect to demographic and other baseline variables. The distribution of clinical response at the post-treatment assessments was analysed using log-linear models to take account of stratification, which included the terms for the response categories, treatments allocated and infection. Comparisons of cure and improvement rates were carried out similarly. The odds ratio for clinical response, which illustrates a measure of the odds in favour of being cured or improved using ceftazidime, as opposed to using imipenem/cilastatin was calculated. In addition, each infection stratum was examined separately for the cure and improvement rates using the chi-squared test. Relapse rates in those initially cured or improved were compared within each stratum using continuity corrected chi-squared test. In the case of small numbers of relapse rates (less than five) occurring for each of the treatments within each stratum Fisher's Exact test was applied. Bacteriological clearance rates were compared between treatment groups for all three strata, using chisquared test and in some cases Fisher's Exact test. Log-linear models were used to compare the treatment groups for the septicaemia and pneumonia strata. An odds ratio for the bacteriological response in pseudomonal and staphylococcal pneumonia was then calculated. Superinfection rates were compared between treatment groups using chi-squared test. The treatment groups were compared, within stratum, with respect to incidence of adverse events using Fisher's Exact Test.

\section{Results}

\section{Patient population}

Three hundred and ninety-three patients were recruited, of which 196 received ceftazidime and 197 imipenem/cilastatin. The two groups were well balanced with respect to age, sex, type of infection, duration of treatment, prognosis of underlying disease, and numbers in intensive care and on assisted ventilation (Table I). The APACHE II scores assessed in 344 patients clearly demonstrated that patients with pneumonia or septicaemia were more seriously ill at the start of treatment than patients with UTI. Forty-six per cent of patients in each arm had received previous antibiotic treatment.

Forty-two patients on ceftazidime and 35 on imipenem/cilastatin were considered to be clinically unevaluable. Reasons were failure to meet protocol definition of nosocomial infection ( 32 on ceftazidime and 25 on imipenem/cilastatin), concurrent treatment with other antimicrobials (eight patients on ceftazidime, three on imipenem/cilastatin) and death due to underlying disease (two patients on ceftazidime, seven on imipenem/ cilastatin). Patients were omitted from the bacteriologically efficacy analysis if either specimens failed to give a pathogen before the start of treatment or there were no valid pre- or post-treatment cultures.

\section{Clinical response}

There were no significant differences between the distribution of clinical responses of the two treatments for all patients $(P=0.65)$ and for evaluable patients only $(P=$ 
Table I. Patient characteristics

\begin{tabular}{|c|c|c|}
\hline Characteristic & ceftazidime & $\begin{array}{l}\text { Treatment } \\
\quad \text { imipenem/cilastin }\end{array}$ \\
\hline $\begin{array}{l}\text { Number of patients entered } \\
\text { pneumonia } \\
\text { septicaemia } \\
\text { renal/urinary infection }\end{array}$ & $\begin{array}{r}196 \\
125 \\
47 \\
24\end{array}$ & $\begin{array}{r}197 \\
129 \\
44 \\
24\end{array}$ \\
\hline Sex (male/female) & $122 / 74$ & $129 / 68$ \\
\hline Mean age (years; range) & $58(18-92)$ & $58(16-90)$ \\
\hline $\begin{array}{l}\text { Prognosis of underlying disease } \\
\text { non-fatal } \\
\text { ultimately fatal } \\
\text { rapidly fatal } \\
\text { unknown }\end{array}$ & $\begin{array}{r}100(51 \%) \\
82(42 \%) \\
12(6 \%) \\
2\end{array}$ & $\begin{array}{c}97(49 \%) \\
78(40 \%) \\
21(11 \%) \\
1\end{array}$ \\
\hline $\begin{array}{l}\text { Number of patients in intensive care units } \\
\text { (\% of entered) }\end{array}$ & $142(72 \%)$ & $143(73 \%)$ \\
\hline $\begin{array}{l}\text { Number of patients on assisted ventilation } \\
\text { ( } \% \text { of entered) }\end{array}$ & $97(49 \%)$ & $100(51 \%)$ \\
\hline 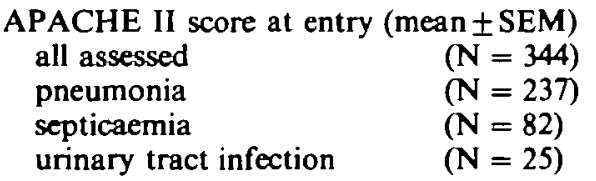 & $\begin{array}{r}13.8 \pm 0.5 \\
13.6 \pm 0.6 \\
15 \cdot 7 \pm 1 \cdot 1 \\
9.4 \pm 1 \cdot 5\end{array}$ & $\begin{array}{l}14 \cdot 1 \pm 0.5 \\
14 \cdot 4 \pm 0.5 \\
14 \cdot 1 \pm 0.9 \\
11 \cdot 5 \pm 2 \cdot 2\end{array}$ \\
\hline Mean duration of treatment (days; SD) & $8 \cdot 8 \pm 3 \cdot 6$ & $9 \cdot 7 \pm 4 \cdot 0$ \\
\hline
\end{tabular}

$0 \cdot 62$ ). The clinical cure plus improvement rates were also not found to be significantly different between treatment groups, both including and excluding unevaluable patients (Table II).

The odds in favour of an infection being cured or improved by ceftazidime as opposed to imipenem/cilastatin was found not to be significant, as illustrated by the $95 \%$ confidence interval of the odds ratio covering $1(0 \cdot 6,1 \cdot 4)$.

When the individual chi-squared tests (both including and excluding unevaluable patients) for each infection stratum were examined, again there were no significant differences between treatment groups.

The percentage of clinically cured or improved patients who experienced clinical relapse during the follow-up period was not significantly different between the two groups for any of the three strata (for pneumonia: ceftazidime 13\%, imipenem/

Table II. Clinical response to treatment

\begin{tabular}{lcccccc}
\hline & \multicolumn{2}{c}{ Pneumonia } & \multicolumn{2}{c}{ Septicaemia } & \multicolumn{2}{c}{ UTI } \\
Response & CAZ & IMI & CAZ & IMI & CAZ & IMI \\
& $(n=125)$ & $(n=129)$ & $(n=47)$ & $(n=44)$ & $(n=24)$ & $(n=24)$ \\
\hline Cured & $45 \%$ & $49 \%$ & $53 \%$ & $59 \%$ & $75 \%$ & $75 \%$ \\
Improved & $18 \%$ & $14 \%$ & $0 \%$ & $0 \%$ & $8 \%$ & $8 \%$ \\
Failure & $18 \%$ & $22 \%$ & $19 \%$ & $14 \%$ & $0 \%$ & $4 \%$ \\
Unevaluable & $19 \%$ & $15 \%$ & $28 \%$ & $27 \%$ & $16 \%$ & $13 \%$ \\
\hline
\end{tabular}

CAZ, Ceftazidime; IMI, imipenem/cilastatin. 
Table III. Pre-treatment pathogens

\begin{tabular}{|c|c|c|c|c|c|c|}
\hline & \multicolumn{6}{|c|}{ Type of infection and treatment } \\
\hline & \multicolumn{2}{|c|}{ pneumonia } & \multicolumn{2}{|c|}{ septicaemia } & \multicolumn{2}{|c|}{ UTI } \\
\hline & CAZZ & IMI & CAZ & IMI & CAZ & IMI \\
\hline Number of patients & 57 & 62 & 30 & 33 & 24 & 24 \\
\hline Number of isolates & 76 & 82 & 35 & 35 & 27 & 30 \\
\hline \multicolumn{7}{|l|}{ Pathogens: } \\
\hline S. aureus & 18 & 16 & 3 & 4 & 0 & 0 \\
\hline Staphylococcus spp. & 0 & 0 & 3 & 1 & 1 & 3 \\
\hline Streptococcus spp. & 4 & 3 & 2 & 2 & 1 & 4 \\
\hline Haemophilus spp. & 8 & 8 & 0 & 0 & $\mathbf{0}$ & 0 \\
\hline E. coli & 9 & 12 & 6 & 7 & 15 & 6 \\
\hline Klebsiella spp. & 7 & 6 & 7 & 5 & 4 & 3 \\
\hline Enterobacter spp. & 2 & 4 & 1 & 3 & 0 & 3 \\
\hline Proteus spp. & 4 & 4 & 1 & 2 & 2 & 2 \\
\hline Acinetobacter spp. & 4 & 3 & 1 & 1 & 0 & 0 \\
\hline Serratia spp. & 2 & 2 & 2 & 0 & 1 & 1 \\
\hline Citrobacter spp. & 1 & 2 & 0 & 0 & 0 & 1 \\
\hline$P$. aeruginosa & 16 & 14 & 8 & 6 & 2 & 6 \\
\hline Psudomonas spp. & 1 & 5 & 0 & 2 & 1 & 0 \\
\hline Other Gram-negatives & 0 & 3 & 1 & 2 & 0 & 1 \\
\hline
\end{tabular}

CAZ, Ceftaridime; IMI, imipenem/cilastatin.

cilastatin $17 \%$, for septicaemia: ceftazidime $8 \%$, imipenem/cilastatin $8 \%$ and for UTI: ceftazidime $25 \%$, imipenem/cilastatin $40 \%$ ).

Finally, no significant difference was found in the distribution of superinfection rates between the two treatment groups (ceftazidime 9\%, imipenem/cilastatin $5 \%$ ). The superinfecting organisms were $S$. aureus, coagulase-negative staphylococci, Enterobacter spp., Klebsiella pneumoniae, P. aeruginosa, Candida spp. and Aspergillus spp. in ceftazidime treated patients; and coagulase-negative staphylococci, Pseudomonas spp., Enterobacter spp., $K$. pneumoniae, and Candida spp. in those receiving imipenem/cilastatin. In both groups Enterococcus faecalis was the main superinfecting organism in the urinary treat.

\section{Bacteriological response}

Two hundred and eight-five organisms, considered to be pathogens by the monitoring committee, were isolated pre-treatment from 230 patients (Table III). Gram-negative organisms predominated, the most common being Escherichia coli (19\% of all isolates) and $P$. aeruginosa $(18 \%)$. Among Gram-positive species, $S$. aureus (14\% of all isolates) was the most common. A majority of the patients had single organisms. Overall, there was no significant difference between the two antibiotics in bacteriological response in the individual infection categories. Subgroup analysis in patients with pseudomonal pneumonia (Table IV) showed that significantly more patients failed to respond microbiologically $(P=0-004)$, though not clinically, to imipenem/cilastatin than to ceftazidime. Bacteriological clearance occurred with imipenem/cilastatin in only $54 \%$ of the clinically cured or improved pseudomonal pneumonias, and in none of the clinical failures. The odds in favour of a patient being cleared of pseudomonal pneumonia was eight times greater when using ceftazidime as opposed to using 
Table IV. Outcome in evaluable patients with pseudomonal pneumonia

\begin{tabular}{llcc}
\hline $\begin{array}{l}\text { Clinical } \\
\text { response }\end{array}$ & $\begin{array}{l}\text { Bacteriological } \\
\text { response }\end{array}$ & $\begin{array}{c}\text { Percentage of } \\
\text { CAZ }\end{array}$ & $\begin{array}{c}\text { patients } \\
\text { IMI }\end{array}$ \\
\hline Cured/improved & & $(n=13)$ & $(n=13)$ \\
& Cleared & $85 \%$ & $54 \%$ \\
Failed & Persistence & $15 \%$ & $46 \%$ \\
& & $(n=4)$ & $(n \pm 6)$ \\
& Cleared & $75 \%$ & $0 \%$ \\
& Persistence & $25 \%$ & $83 \%$ \\
& Unassessable & $0 \%$ & $17 \%$ \\
\hline
\end{tabular}

CAZ, Ceftazidime, IMI, imipenem/cilastatin

imipenem/cilastatin (95\% confidence interval 1.7-38). Furthermore, in six of the 11 imipenem/cilastatin patients in whom the organism persisted, resistance to the study drug developed during treatment; this was found in only one patient on ceftazidime.

In patients with pneumonia caused by $S$. aureus, 12 of 18 patients in the ceftazidime group and 12 of 16 in the imipenem/cilastatin group were cured or improved clinically. Persistence of staphylococci was seen in five ceftazidime patients, of whom three were clinical failures. In the imipenem/cilastatin group, only one strain persisted and that patient also failed clinically. This difference was not statistically significant.

A sample of around $35 \%$ of the isolates was sent from the participating study centres to a central laboratory for both identification and determination of their MICs to ceftazidime and imipenem. Results obtained by the study centres and the central laboratory were consistent in about $90 \%$ of cases with respect to the identity of isolates. By conversion of the MICs (from the central laboratory) to categories of susceptible, moderate and resistant (from the study centres), using breakpoint concentrations specified in the NCCLS guidelines, susceptibility results were shown to be consistent in over $70 \%$ of cases. These results indicate a comparable expertise in identification and susceptibility testing throughout the participating centres.

\section{Adverse events}

Overall $42(21 \%)$ patients in the ceftazidime treatment group and $55(28 \%)$ in the imipenem/cilastatin treatment group experienced one or more adverse events. The

Tabłe V. Adverse events considered drug-related

\begin{tabular}{lcc}
\hline Adverse effect & $\begin{array}{c}\text { Treatment and number of patients } \\
\text { CAZ } \\
(n=4)\end{array}$ & $\begin{array}{c}\text { IMI } \\
(n=8)\end{array}$ \\
\hline Rash & 1 & 2 \\
Drug fever & 1 & 0 \\
Phlebitis & 0 & 1 \\
Diarrhoea & 2 & 1 \\
Grand mal seizure & 0 & 1 \\
Candida superinfection (oral/genital) & 0 & 2 \\
Elevated creatinine & 0 & 1 \\
\hline
\end{tabular}

CAZ, Ceftazidime; IMI, imipenem/cilastatin. 
difference in incidence between the two treatment groups was not statistically significant. The majority of adverse events were not considered by the investigators to be drug-related. Only 12 patients (four ceftazidime, eight imipenem/cilastatin) had adverse events which were considered by the investigators to be possibly, probably or almost certainly related to antibiotic treatment (Table V). Treatment was discontinued due to adverse events in three patients; two on ceftazidime, and one on imipenem/cilastatin. One patient on imipenem/cilastatin experienced a single grand mal seizure on each of two consecutive days which resolved, with no further recurrence, after a decrease in the dose.

Thirty-one of the patients receiving ceftazidime died during treatment, or within the 14 day follow-up period. Three of these died during treatment due to bacterial sepsis. This compares with 20 and four patients respectively on imipenem/cilastatin. All deaths were considered unrelated to the study drugs.

\section{Discussion}

This study demonstrates that ceftazidime and imipenem/cilastatin are both effective as monotherapy in seriously ill patients with nosocomial infections. There were no significant differences between ceftazidime and imipenem/cilastatin in clinical efficacy: the proportion of evaluable patients receiving ceftazidime who were cured or improved ranged from 74 to $100 \%$ depending on the infection, and from 74 to $95 \%$ for those given imipenem/cilastatin. The frequency of isolation of individual pathogens in this study was similar to other reports in the literature (Horan et al., 1988; Scheld \& Mandell, 1991).

In patients with pneumonia $23 \%$ of pathogens isolated were $P$. aeruginosa or other Pseudomonas spp. Significantly more of these organisms persisted in patients who were given imipenem/cilastatin than in those who received ceftazidime, but this was not always associated with clinical failure. This suggests that in some patients the $P$. aeruginosa strains may not have been the causative agents. One possible explanation for the development of resistance to imipenem/cilastatin seen in around half of the persistent isolates in this study is that the $0.5 \mathrm{~g}$ dose of imipenem/cilastatin study gives plasma concentrations above the minimum inhibitory concentration for $P$. aeruginosa for about $2.5 \mathrm{~h}$. This relatively low dose could have resulted in the development, during treatment, of imipenem-resistant $P$. aeruginosa, a phenomenon which has been reported previously (Acar, 1985; Krilov et al., 1985; Salata et al., 1985). It is of interest that the principal resistance mechanism in $P$. aeruginosa to these compounds is different. With ceftazidime, resistance is generally associated with $\beta$-lactamase production, which may also promote variable imipenem/cilastatin resistance. Imipenem/cilastatin resistance, on the other hand, derives from altered permeability via a protein change (Trias \& Nikaido, 1990): the protein D2 which is responsible for specific transport of imipenem into $P$. aeruginosa is not present in resistant isolates.

This large trial has emphasized certain points: the APACHE II scoring system proved reliable in the classification of the seriousness of disease and for stratifying the degree of risk of subsequent death. It indicated that the patients with UTI were less seriously ill, but excluding these patients did not alter the overall outcome. With the fixed sample size chosen the high response rates seen with both treatments in patients with severe UTI could have reduced substantially the capacity to detect a difference in outcome in the two more serious conditions of pneumonia or septicaemia. In future 
studies of nosocomial infections, severe UTI should be evaluated in a separate protocol.

In the patients with pneumonia the protocol definition was adhered to and cases of purulent tracheobronchitis were excluded from the evaluation of efficacy, although many authorities recognize that this group of patients (particularly those with $P$. aeruginosa in tracheal aspirates) warrant antibiotic treatment. Likewise, cases of ARDS (19 in all) were excluded from clinical evaluation unless an infectious complication such as empyema or a superinfection in the blood developed.

Again, in cases of pneumonia in the Intensive Care Unit bacteriological confirmation is imprecise. The upper airways of patients are commonly colonized with potential respiratory pathogens, with the result that organisms obtained from tracheal suction or sputum are not necessarily diagnostic. In this study respiratory specimens came from a variety of sources, but the pathogens isolated were consistent with a nosocomial pattern of infection.

In addition, in patients with pneumonia and in whom $P$. aeruginosa was isolated, there was a clear correlation between failure to eradicate these organisms and poor clinical outcome, especially in the imipenem/cilastatin group. Of course, bacteriological diagnosis can be improved by the use of a fibre-optic bronchoscope with either broncho-alveolar lavage or the use of the protected specimen brush (Thorpe et al., 1987; Faling, 1988; Fagon et al., 1988). Quantitative culture will enhance the specificity of both methods, and it seems appropriate to recommend the use of these techniques for future studies in this population of patients.

In conclusion, this trial has demonstrated that single agent treatment can given satisfactory results in severe hospital acquired infections, including pseudomonal pneumonia, in non-neutropenic patients. The choice of antibiotic in an individual hospital or unit should be made after the consideration of several factors, including local sensitivity patterns.

\section{Acknowledgements}

This study was supported by a grant from Glaxo Group Research Ltd, Greenford, UK.

\section{References}

Acar, J. F. (1985). Therapy for lower respiratory tract infections with imipenem/cilastatin: a review of worldwide experience. Reviews of Infectious Diseases 7, Suppl. 3, S513-7.

Carpenter, J. L. (1990). Klebsiella pulmonary infections: occurrence at one medical center and review. Reviews of Infectious Diseases 12, 672-2.

Clissold, S. P. Todd, P. A. \& Campoli-Richards, D. M. (1987). Imipenem/cilastatin. A review of its antibacterial activity, pharmacokinetic properties and therapeutic efficacy. Drugs 33, 183-241.

Fagon, J. Y., Chastre, J., Trouillet, J. L., Domart, Y., Scheimberg, A., Darne, C. et al. (1988). Comparison of clinical assessment and invasive procedure in the evaluation of patients suspected of having nosocomial pneumonia. Intensive Care Medicine 14, Suppl. I, 263.

Faling, L. J. (1988). New advances in diagnosing nosocomial pneumonia in intubated patients. Part I. American Review of Respiratory Disease 137, 253-5.

Gardner, W. G. (1983). Multicentered clinical evaluation of cefoperazone for the treatment of lower respiratory tract infections. Reviews of Infectious Diseases 5, S137-44.

Horan, T., Culver, D., Jarvis, W., Emori, G., Banerjee, S., Martoni, W. et al. (1988). Pathogens causing nosocomial infections. Preliminary data from the national nosocomial infections surveillance system. Antimicrobial Newsletter 5, 65-7. 
Knaus, W. A., Draper, E. A., Wagner, D. P. \& Zimmerman, J. E. (1985). APACHE II: A severity of disease classification system. Critical Care Medicine 13, 818-29.

Krilov, L. R., Blumer, J. L., Stern, R. C., Hartstein, A. I. Iglewski, B. N. \& Goldmann, D. A. (1985). Imipenem/cilastatin in acute pulmonary exacerbations of cystic fibrosis. Reviews of Infectious Diseases 7, Suppl. 3, S482-9.

Laforce, F. M. (1989). Systemic antimicrobial therapy of nosocomial pneumonia: monotherapy versus combination therapy. European Journal of Clinical Microbiology and Infectious Diseases 8, 61-8.

Ledingham, I. M., Alcock, S. R., Eastaway, A. T., McDonald, J. C., McKay, I. C. \& Ramsay, G. (1988). Triple regimen of selective decontamination of the digestive tract, systemic cefotaxime, and microbiological surveillance for prevention of acquired infection in intensive care. Lancet $i, 785-90$.

Moore, R. D., Smith, C. R. \& Leitman, P. S. (1984). Association of aminoglycoside plasma levels with therapeutic outcome in Gram-negative pneumonia. American Journal of Medicine 77 , 657-62.

Salata, R. A., Gebhart, R. L., Palmer, D. L., Wade, B. H., Scheld, W. M., Groschel, D. H. M. et al. (1985). Pneumonia treated with imipenem/cilastatin. American Journal of Medicine 78, Suppl. 6A, 104-9.

Sanders, J. W., Powe, N. R. \& Moore, R. D. (1991). Ceftazidime monotherapy for empiric treatment of febrile neutropenic patients: a metaanalysis. Journal of Infectious Diseases 164, 907-16.

Scheld, W. M. \& Mandell, G. L. (1991). Nosocomial pneumonia: pathogenesis and recent advances in diagnosis and therapy. Reviews of Infectious Diseases 13, Suppl. 9, S743-51.

Stoutenbeek, C. P., van Saene, H. K. F., Miranda, D. R. Zandstra, D. F. \& Langrehr, D. (1987). The effect of oropharyngeal deconamination using topical nonabsorbable antibiotics on the incidence of nosocomial respiratory tract infections in multiple trauma patients. Journal of Trauma 27, 357-64.

Thorpe, J. E., Baughman, R. P., Frame, P. T., Wesseler, T. A. \& Staneck, J. L. (1987). Bronchoalveolar lavage for diagnosing acute bacterial pneumonia. Journal of Infectious Diseases 155, Suppl. 5, 855-61.

Trias, J. \& Nikaido, H. (1990). Outer membrane protein D2 catalyzes facilitated diffusion of carbapenems and penems through the outer membrane of Pseudomonas aeruginosa. Antimicrobial Agents and Chemotherapy 34, 52-7. 